\title{
Anhydrous (Non-Cyrogenic) Specimen Preparation of Biological Samples for Energy Dispersive Spectroscopy in the Transmission Electron Microscope.
}

\author{
E. Ann Ellis ${ }^{1}$ and Lynn Blubaugh ${ }^{2}$ \\ ${ }^{1}$ Microscopy and Imaging Center, Texas A\&M University, College Station, TX \\ ${ }^{2}$ Hitachi High Technologies-America, Gaithersburg, MD
}

Elemental analysis of biological materials is often challenging as a result of loss of elements during specimen preparation in the aqueous solvents used in both fixation, dehydration and embedding. We developed an anhydrous specimen preparation technique for preparing pieces of lichen tissue for energy dispersive spectroscopy (EDS) of soluble element' specimens of lichens have been examined by EDS in the scanning electron microscope. In addition, freeze drying and lyophilization have been done for similar samples with varying quality and reproducibility of results. This paper presents a modification of earlier work by Landis [1] for anhydrous preparation of TEM specimens.

Pieces of lichens were collected from newly fallen oak branches after several days of rehydration by rain. The anhydrous fixation protocol was modified from the work of Landis [1]. Small (1-2 mm) pieces of the lichen thallus were cut with a scalpel and placed in a petri dish in the hood. The lichen was fixed by acrolein vapors from acrolein in a small plastic lid placed near the tissue. A top was placed on the glass petri dish and a beaker of hot water was placed on the top of the petri dish. Additional acrolein was added after several hours and the vapor chamber was left overnight. Pieces of lichen were then transferred to a scintillation vial which contained $1 \mathrm{ml}$ of anhydrous (stored over molecular sieves) ethylene glycol for $24 \mathrm{hrs}$ on a low speed rotator at room temperature. The ethylene glycol was replaced with Quetol 651 (Ladd Research, Williston, VT), a low viscosity, alkane epoxy resin, and the vial was returned to the rotator. After several changes of Quetol 651, the specimens were slowly infiltrated with a low viscosity epoxy resin [Quetol 6511.74 g, ERL 42212.07 g, NSA 6.19 g, DER 7361.0 g, and BDMA $0.25 \mathrm{ml}$ ] over a period of four days on a low speed rotor at room temperature. Infiltration was assisted by intermittent vacuum and microwave cycle of $6 \min (2 \operatorname{min~ON;} 2 \min O F F ; 2 \min O N)$ at 250 watts. New epoxy resin was made at each resin change and for final embedding. Sections were cut onto ethylene glycol or deionized water and picked up on 200 mesh grids. Grids were stabilized with 10 $\mathrm{nm}$ of carbon and then imaged and analyzed in a Hitachi 7700 TEM at $100 \mathrm{kV}$.

Unstained thick sections showed detail of the lichen ultrastructure (Fig. 1) and EDS maps (Fig. 2,3) demonstrated a number of elements throughout the tissue. In the past, only EDS analysis of bulk samples of lichens by SEM has been published. Many tissues do not lend themselves to high pressure freezing because of self-insulation and varying water content which results in ice crystal damage. The advantage of this specimen preparation technique is that no complicated equipment is needed and the problem of ice crystal formation is avoided. In the event that infiltration is not suitable, it is possible to extend the times for all steps of the preparation. Using smaller pieces of sample is another alternative to improve specimen preparation.

The ultrastructure and contrast from this preparative procedure and elemental analysis demonstrated the presence of common elements in the environment such as phosphorus, sulfur, and magnesium (identified in a previous spectrum) (Figs. 1-3). In STEM, the higher atomic weight elements appear bright. 


\section{References}

[1] WJ Landis J. et al, J. Ultrastruct. Res. 70(1979), p.171.

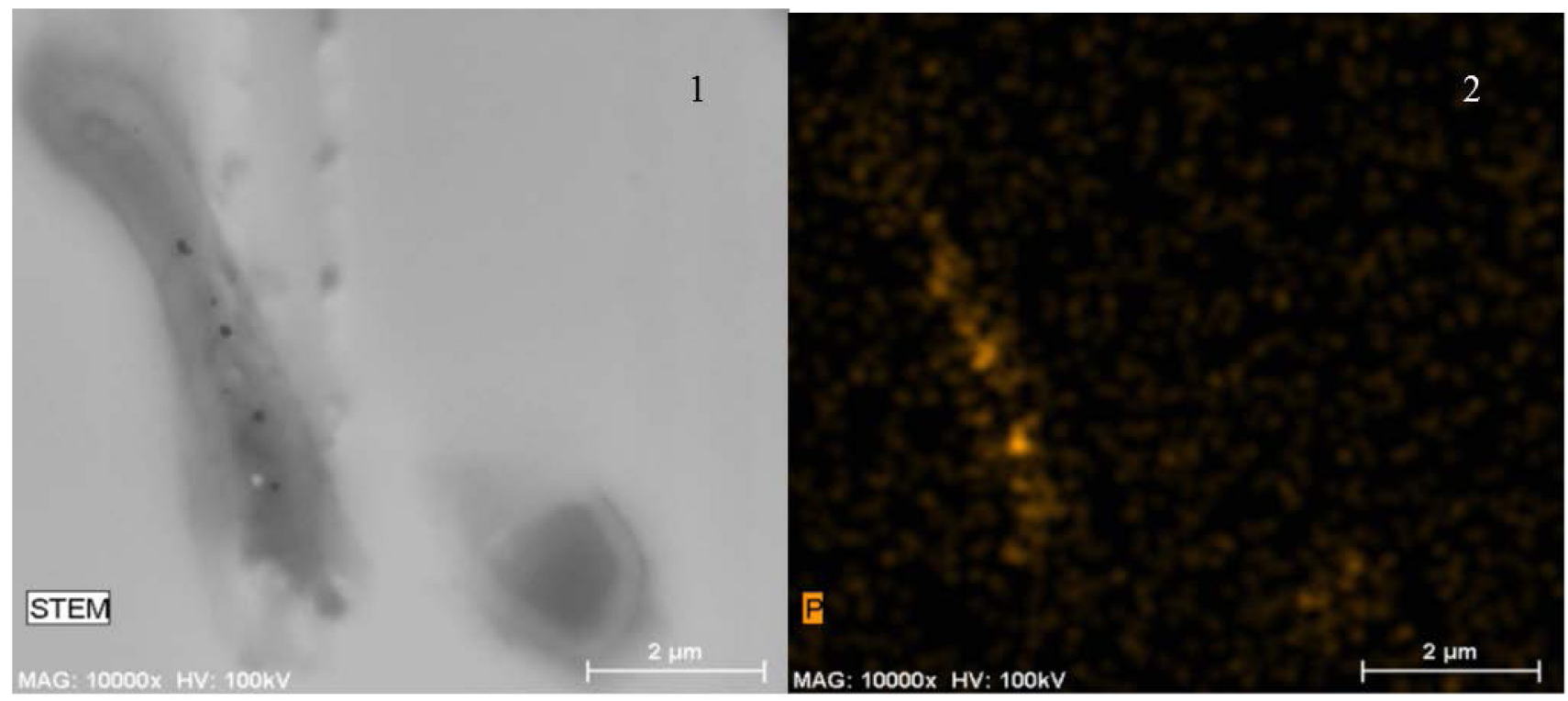

Fig. 1 STEM image of section through a lichen thallus. The light areas have a higher atomic weight. Fig. 2 Map of phosphorus (orange) in the same section as shown in Fig. 1.

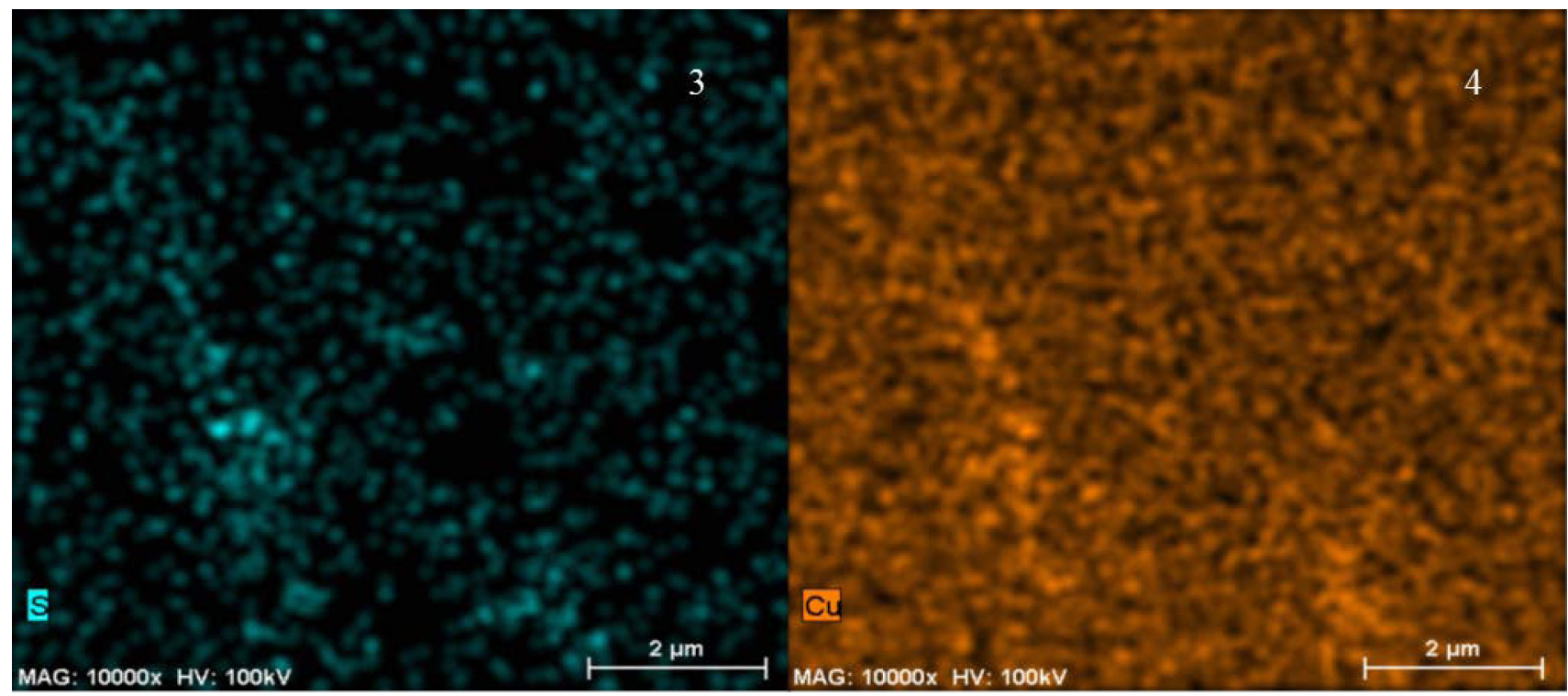

Fig. 3 Map of sulfur (blue) (bright areas) in the same section as shown in Figs. 1 and 2. Fig. 4 Map of copper (bright areas) some of which may come from the grid. 